\title{
Study of Heat Transport in a Porous Medium Under G-jitter and Internal Heating Effects
}

\author{
B. S. Bhadauria - I. Hashim • P. G. Siddheshwar
}

Received: 17 July 2012 / Accepted: 17 August 2012 / Published online: 5 September 2012

(C) Springer Science+Business Media B.V. 2012

\begin{abstract}
In this article we study the combined effect of internal heating and time-periodic gravity modulation on thermal instability in a closely packed anisotropic porous medium, heated from below and cooled from above. The time-periodic gravity modulation, considered in this problem can be realized by vertically oscillating the porous medium. A weak non-linear stability analysis has been performed by using power series expansion in terms of the amplitude of gravity modulation, which is assumed to be small. The Nusselt number has been obtained in terms of the amplitude of convection which is governed by the non-autonomous Ginzburg-Landau equation derived for the stationary mode of convection. The effects of various parameters such as; internal Rayleigh number, amplitude and frequency of gravity modulation, thermo-mechanical anisotropies, and Vadász number on heat transport has been analyzed. It is found that the response of the convective system to the internal Rayleigh number is destabilizing. Further it is found that the heat transport can also be controlled by suitably adjusting the external parameters of the system.
\end{abstract}

Keywords Non-linear stability analysis · Ginzburg-Landau equation ·

Gravity modulation $\cdot$ Internal heating

\footnotetext{
B. S. Bhadauria $(\varangle)$

Department of Applied Mathematics, School for Physical Sciences, Babasaheb Bhimrao Ambedkar University, Lucknow 226 025, India

e-mail: mathsbsb@yahoo.com

I. Hashim

School of Mathematical Sciences, Faculty of Science and Technology,

Universiti Kebangsaan Malaysia, 43600 Bangi, Selangor, Malaysia

e-mail: ishak_h@ukm.my

P. G. Siddheshwar

Department of Mathematics, Bangalore University, Central College Campus, Bangalore 560 001, India e-mail:pgsmath@gmail.com
} 


\section{List of Symbols}

\section{Variables}

$\begin{array}{ll}A & \text { Amplitude of convection } \\ d & \text { Height of the fluid layer } \\ D a & \text { Darcy number } D a=K_{z} / \mathrm{d}^{2} \\ g_{0} & \text { Mean of gravity } \\ Q & \text { Internal heat source } \\ k_{\mathrm{c}} & \text { Critical wave number } \\ N u & \text { Nusselt number } \\ p & \text { Reduced pressure } \\ P r & \text { Prandtl number, } P r=v / \kappa_{z} \\ R a & \text { Thermal Rayleigh number, } R a=\alpha_{T} g_{0} K_{z}(\Delta T) \mathrm{d} / \nu \kappa_{T z} \\ R_{0 \mathrm{c}} & \text { Critical Rayleigh number } \\ R_{\mathrm{i}} & \text { Internal Rayleigh number, } R_{\mathrm{i}}=Q \mathrm{~d}^{2} / \kappa_{T z} \\ V a & \text { Vadász number, } V a=\phi P r / D a \\ \xi & \text { Mechanical anisotropy parameter } \xi=K_{x} / K_{z} \\ \eta & \text { Thermal anisotropy parameter } \eta=\kappa_{T x} / \kappa_{T z} \\ t & \text { Time } \\ T & \text { Temperature } \\ \Delta T & \text { Temperature difference across the fluid layer } \\ x, y, z & \text { Space co-ordinates }\end{array}$

\section{Greek Symbols}

$\alpha_{T} \quad$ Coefficient of thermal expansion

$\delta^{2} \quad$ Horizontal wave number $k_{\mathrm{c}}^{2}+\pi^{2}$

$\delta_{1} \quad$ Amplitude of temperature modulation

$\Omega \quad$ Frequency of modulation

$\epsilon \quad$ Perturbation parameter

$\gamma$ Heat capacity ratio $\frac{\left(\rho c_{p}\right)_{m}}{\left(\rho c_{p}\right)_{f}}$

K Permeability tensor

$\kappa_{T} \quad$ Effective thermal diffusivity

$\mu \quad$ Effective dynamic viscosity of the fluid

$v$ Effective kinematic viscosity, $\left(\frac{\mu}{\rho_{0}}\right)$

$\phi$ Porosity

$\psi \quad$ Stream function

$\rho \quad$ Fluid density

$\tau \quad$ Slow time $\tau=\epsilon^{2} t$

\section{Other Symbols}

$$
\begin{array}{ll}
\nabla^{2} & \frac{\partial^{2}}{\partial x^{2}}+\frac{\partial^{2}}{\partial y^{2}}+\frac{\partial^{2}}{\partial z^{2}} \\
\nabla_{1}^{2} & \frac{\partial^{2}}{\partial x^{2}}+\frac{\partial^{2}}{\partial z^{2}} \\
\nabla_{\xi}^{2} & \frac{\partial^{2}}{\partial x^{2}}+\frac{1}{\xi} \frac{\partial^{2}}{\partial z^{2}} \\
\nabla_{\eta}^{2} & \eta \frac{\partial^{2}}{\partial x^{2}}+\frac{\partial^{2}}{\partial z^{2}}
\end{array}
$$




\section{Subscripts \\ b Basic state \\ c Critical \\ 0 Reference value}

\author{
Superscripts \\ , Perturbed quantity \\ * Dimensionless quantity \\ st Stationary
}

\title{
1 Introduction
}

The study of thermal instability in a fluid saturated horizontal porous medium has received a considerable attention due to its numerous applications in many practical problems. It has a wide range of applications in geophysics, oil recovery process, in petroleum industry, and in solidification of polymeric liquids. A comprehensive account of many studies on thermal instability in porous media and their applications in various fields is given in the excellent books of Ingham and Pop (1998), Ingham and Pop (2005), Vafai (2000), Vafai (2005), Straughan (2004), Nield and Bejan (2006), and Vadász (2008).

There are many practically important situations, where the porous material offers its own source of heat. This gives a different way in which a convective flow can be set up through the local heat generation within the porous media. Such a situation can occur through radioactive decay or through, in the present perspective, a relatively weak exothermic reaction which can take place within the porous material. To be more specific, internal heat is the main source of energy for celestial bodies caused by nuclear fusion and decaying of radioactive materials, which keeps the celestial objects warm and active. It is due to the internal heating of the earth that there exists a thermal gradient between the interior and exterior of the earth's crust, saturated by multicomponents fluids, which helps convective flow, thereby transferring the thermal energy towards the surface of the earth. Therefore, the role of internal heat generation becomes very important in several applications that include geophysics, reactor safety analyses, metal waste form development for spent nuclear fuel, fire and combustion studies, and storage of radioactive materials. However, there are relatively very few studies available in which the effect of internal heating on convective flow has been investigated. Some of these studies are; Bhattacharya and Jena (1984), Haajizadeh et al. (1984), Rionero and Straughan (1990), Rao and Wang (1991), Parthiban and Patil (1997), Herron Isom (2001), Khalili and Huettel (2002), Joshi et al. (2006), Bhadauria et al. (2011), and Bhadauria (2012).

The gravity modulation of the system leads to the variable coefficients in the governing equations of thermal instability in porous media and involves the vertical time-periodic vibrations of the system. Further, this leads to the appearance of a modified gravity, collinear with actual gravity, in the form of a time-periodic gravity field perturbation and is known as gravity modulation or G-jitter in literature. Some of the documented works on gravity modulation in recent times include Yang (1997), Malashetty and Padmavathi (1997), Rees and Pop (2000, 2001, 2003), Malashetty and Basavaraj (2002), Govender (2004, 2005a,b), Kuznetsov (2005, 2006a,b), Siddhavaram and Homsy (2006), Strong (2008a,b), Razi et al. (2009), Saravanan and Purusothaman (2009), Vanishree (2010), Saravanan and Arunkumar (2010), Malashetty and Swamy (2011), and Saravanan and Sivakumar (2010, 2011), Siddheshwar et al. (2012). 
However, most of the studies on thermal instability in a porous medium with gravity modulation concern only linear stability and hence address only questions on onset of convection. The linear stability analysis is inadequate and the nonlinear stability analysis becomes inevitable if one wants to consider the heat transport in the system. Recently Siddheshwar et al. (2012) have studied the heat transport in Bénard-Darcy convection with G-jitter and thermomechanical anisotropy in variable viscosity liquids.

Anisotropy in porous media, which is due to the preferential orientation or asymmetric geometry of porous matrix or fibres, is encountered in many systems in industry and nature, and in the present context, is of particular interest in the study of extraction of metals from ores where a mushy layer is formed during solidification of a metallic alloy. Further, the quality and structure of the resulting solid can be controlled by influencing the transport process. Since, internal heating in porous media or gravity modulation of the system or a combination of both is an effective mechanism to control the convective flow by suppressing or advancing it, therefore they can be used as external means to influence the quality and structure of the resulting solid. With this motive we have made a weak non-linear analysis of thermal instability in a anisotropic porous medium under gravity modulation, and studied the effect of internal heating on heat transport, using the Ginzburg-Landau equation and in the process quantify the heat transport in terms of the amplitude governed by the Ginzburg-Landau equation.

\section{Mathematical Formulation of the Problem}

We consider an infinitely extended horizontal porous layer saturated with an incompressible viscous Boussinesq fluid confined between two parallel planes, lower plane at $z=0$ and upper one at $z=d$. A Cartesian frame of reference is chosen in such a way that the origin lies on the lower plane and the $z$ axis as vertically upward. The porous layer is heated from below and cooled from above. A schematic diagram of the problem is shown in the Fig. 1. The porous medium is considered to be anisotropic and follow local thermal equilibrium assumption. The Darcy law and the Oberbeck-Boussinesq approximation (Rajagopal et al. 2010) are taken to be applicable. Under these assumption, the equations (Nield and Bejan 2006) which describe the system are given by:

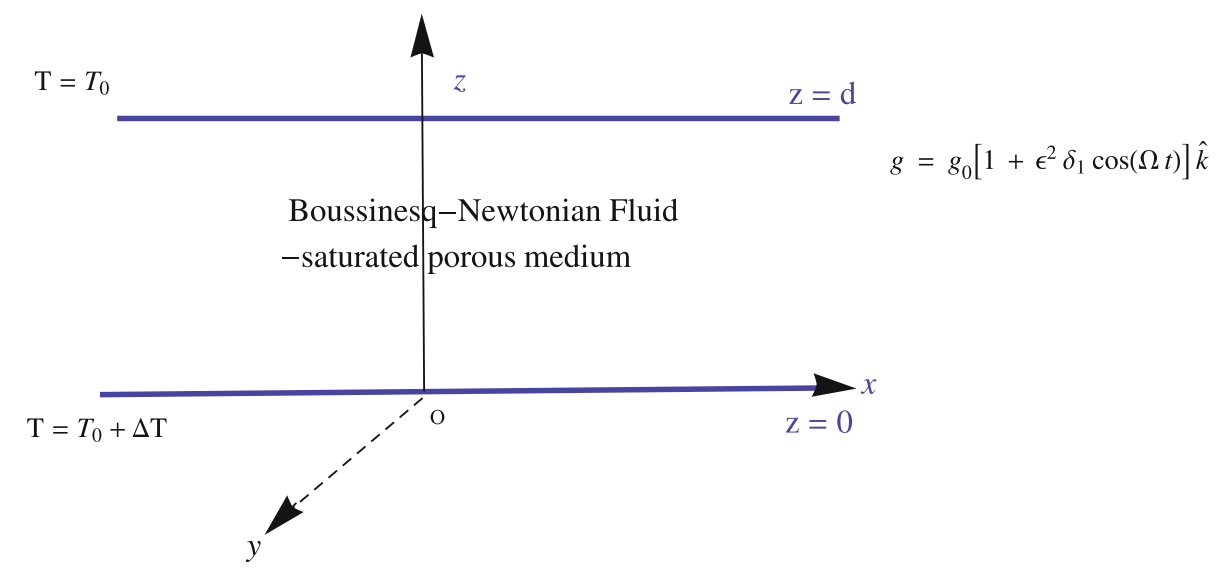

Fig. 1 Physical configuration of the gravity modulation problem 


$$
\begin{aligned}
\nabla \cdot \mathbf{q} & =0 \\
\frac{\rho_{0}}{\phi} \frac{\partial \mathbf{q}}{\partial t} & =-\nabla p+\rho \mathbf{g}(t)-\mu(\mathbf{K} \cdot \mathbf{q}) \\
\gamma \frac{\partial T}{\partial t}+(\mathbf{q} \cdot \nabla) T & =\nabla \cdot\left(\kappa_{T} \cdot \nabla T\right)+Q\left(T-T_{0}\right) \\
\rho & =\rho_{0}\left[1-\alpha_{T}\left(T-T_{0}\right)\right] \\
\mathbf{g}(t) & =g_{0}\left[1+\epsilon^{2} \delta_{1} \cos (\Omega t)\right] \hat{k},
\end{aligned}
$$

where $\mathbf{q}$ is velocity $(u, v, w), \mu$ is the dynamic viscosity, $Q$ is internal heat source, $\mathbf{K}$ is the permeability tensor, $\frac{1}{K_{x}}(\hat{i} \hat{i}+\hat{j} \hat{j})+\frac{1}{K_{z}}(\hat{k} \hat{k}), \kappa_{T}$ is the thermal diffusivity tensor, $\kappa_{T_{x}}(\hat{i} \hat{i}+$ $\hat{j} \hat{j})+\kappa_{T_{z}}(\hat{k} \hat{k}), T$ is temperature, $\alpha_{T}$ is thermal expansion coefficient, $\gamma$ is the ratio of heat capacities, $\rho$ is the density, while $\rho_{0}$ is the reference density. $g_{0}$ is the mean gravity, $\delta_{1}$ is the small amplitude of gravity modulation, $\Omega$ is the frequency of gravity modulation, $\epsilon$ is a quantity that indicates smallness in order of magnitude of modulation and $t$ is the time.

The considered thermal boundary conditions are:

$$
T=T_{0}+\Delta T \text { at } z=0 \text { and } T=T_{0} \text { at } z=d .
$$

Assuming the basic state to be quiescent, the quantities at the basic state are given by,

$$
\mathbf{q}_{\mathrm{b}}=(0,0,0), p=p_{\mathrm{b}}(z), T=T_{\mathrm{b}}(z), \quad \text { and } \rho=\rho_{\mathrm{b}}(z) .
$$

The basic state pressure field is not required here, however, the basic temperature field is governed by the following ordinary differential equation:

$$
\kappa_{T} \frac{\mathrm{d}^{2}\left(T_{\mathrm{b}}-T_{0}\right)}{\mathrm{d} z^{2}}+Q\left(T_{\mathrm{b}}-T_{0}\right)=0,
$$

where "b" refers the basic state. The Eq. (2.8) is solved for $T_{\mathrm{b}}(z)$ subject to the boundary condition (2.6), we get

$$
T_{\mathrm{b}}=T_{0}+\Delta T \frac{\sin \sqrt{\left(Q / \kappa_{T}\right)}\left(1-\frac{z}{\mathrm{~d}}\right)}{\sin \sqrt{\left(Q / \kappa_{T}\right)}} .
$$

We assume finite amplitude perturbations on the basic state in the form:

$$
\mathbf{q}=\mathbf{q}_{\mathrm{b}}+\mathbf{q}^{\prime}, \quad T=T_{\mathrm{b}}+T^{\prime}, \quad p=p_{\mathrm{b}}+p^{\prime}, \quad \rho=\rho_{\mathrm{b}}+\rho^{\prime}
$$

where primes denotes the quantities at the perturbations. Substituting Eq. (2.10) in Eqs. (2.12.3), and using Eq. (2.4), the perturbed equations are obtained as

$$
\begin{aligned}
\nabla \cdot \mathbf{q}^{\prime} & =0 \\
\frac{\rho_{0}}{\phi} \frac{\partial \mathbf{q}^{\prime}}{\partial t} & =-\nabla p^{\prime}-\frac{\mu}{\mathbf{K}} \mathbf{q}^{\prime}+\rho_{0} \mathbf{g}(t) \alpha_{T} T^{\prime} \\
\gamma \frac{\partial T^{\prime}}{\partial t}+\left(\mathbf{q}^{\prime} \cdot \nabla\right) T^{\prime}+w^{\prime} \frac{\mathrm{d} T_{\mathrm{b}}}{\mathrm{d} z} & =\kappa_{T_{x}} \nabla_{1}^{2} T^{\prime}+\kappa_{T_{z}} \frac{\partial^{2} T^{\prime}}{\partial z^{2}}+Q T^{\prime}
\end{aligned}
$$

Equations (2.11-2.13) are non-dimensionalized by using the following scales:

$$
\begin{aligned}
\left(x^{\prime}, y^{\prime}, z^{\prime}\right) & =\left(x^{*}, y^{*}, z^{*}\right) \mathrm{d}, \quad t=\frac{\mathrm{d}^{2}}{\kappa_{T}} t^{*}, \quad \mathbf{q}^{\prime}=\frac{\kappa_{T_{z}}}{\mathrm{~d}} \mathbf{q}^{*}, \\
T^{\prime} & =(\Delta T) T^{*}, \quad p^{\prime}=\frac{\mu \kappa_{T z}}{K_{z}} p^{*}, \quad \Omega=\frac{\kappa_{T z}}{\mathrm{~d}^{2}} \Omega^{*} .
\end{aligned}
$$


The non-dimensional equations (dropping the asterisks for simplicity) are obtain as

$$
\begin{aligned}
& \frac{1}{V a} \frac{\partial q}{\partial t}+\mathbf{q}_{\mathrm{a}}=-\nabla p+R a_{T}\left[1+\epsilon^{2} \delta_{1} \cos (\Omega t)\right] T \\
& \gamma \frac{\partial T}{\partial t}+(\mathbf{q} \cdot \nabla) T+w \frac{\mathrm{d} T_{\mathrm{b}}}{\mathrm{d} z}=\left(\eta \nabla_{1}^{2}+\frac{\partial^{2}}{\partial z^{2}}\right) T+R_{i} T
\end{aligned}
$$

where $R a_{T}=\alpha_{T} g_{0} K_{z}(\Delta T) d / \nu \kappa_{T z}$ is thermal Rayleigh number, $R_{i}=Q \mathrm{~d}^{2} / \kappa_{T z}$ is internal Rayleigh number. $V a=\phi P r / D a$ is Vadász number, $\operatorname{Pr}=v / \kappa_{T_{z}}$ is Prandtl number, $D a=K_{z} / \mathrm{d}^{2}$ is Darcy number, $\mathbf{q}_{\mathrm{a}}=\left(\frac{1}{\xi} u, \frac{1}{\xi} v, w\right)$ is the anisotropic modified velocity vector, $\xi=K_{x} / K_{z}$ is the mechanical anisotropy parameter, $\eta=\kappa_{T x} / \kappa_{T z}$ is the thermal anisotropy parameter and $v=\mu / \rho_{0}$ is kinematic viscosity.

The non-dimensional basic temperature $T_{\mathrm{b}}(z)$ which appears in the Eq. (2.16) can be obtained from the expression (2.9) as

$$
\frac{\mathrm{d} T_{\mathrm{b}}}{\mathrm{d} z}=-\frac{\sqrt{R_{i}} \cos \sqrt{R_{i}}(1-z)}{\sin \sqrt{R_{i}}}
$$

We consider only 2D disturbances in our study, and hence the stream function $\psi$ may be introduced in the form:

$$
u=\frac{\partial \psi}{\partial z}, \quad w=-\frac{\partial \psi}{\partial x} .
$$

Then eliminating the pressure term $p$ from Eq. (2.15), the non-dimensional governing equations now have the form:

$$
\begin{aligned}
& {\left[\frac{1}{V a} \frac{\partial}{\partial t}\left(\frac{\partial^{2}}{\partial x^{2}}+\frac{\partial^{2}}{\partial z^{2}}\right)+\left(\frac{\partial^{2}}{\partial x^{2}}+\frac{1}{\xi} \frac{\partial^{2}}{\partial z^{2}}\right)\right] \psi=-R a\left[1+\epsilon^{2} \delta_{1} \cos (\Omega t)\right] \frac{\partial T}{\partial x}} \\
& \left(\gamma \frac{\partial}{\partial t}-\eta \frac{\partial^{2}}{\partial x^{2}}-\frac{\partial^{2}}{\partial z^{2}}-R_{i}\right) T=\frac{\partial \psi}{\partial x} \frac{\mathrm{d} T_{\mathrm{b}}}{\mathrm{d} z}+\frac{\partial(\psi, T)}{\partial(x, z)}
\end{aligned}
$$

To keep the time variation slow, we have rescaled the time $t$ by using the time scale $\tau=\epsilon^{2} t$. Also to keep the parameters at minimal, the value of $\gamma$ is taken equal to 1 .

Now, to study the stationary convection of the system, we write the non-linear Eqs. (2.192.20 ) in the matrix form as given below:

$$
\left[\begin{array}{cr}
\nabla_{\xi}^{2} & R a \frac{\partial}{\partial x} \\
-\frac{\mathrm{d} T_{\mathrm{b}}}{\mathrm{d} z} \frac{\partial}{\partial x}-\left(\nabla_{\eta}^{2}+R_{i}\right)
\end{array}\right]\left[\begin{array}{l}
\psi \\
T
\end{array}\right]=\left[\begin{array}{r}
-\frac{\epsilon^{2}}{V a} \frac{\partial}{\partial \tau} \nabla_{1}^{2} \psi-\epsilon^{2} \delta_{1} \cos (\omega \tau) R a \frac{\partial T}{\partial x} \\
\frac{\partial(\psi, T)}{\partial(x, z)}-\epsilon^{2} \frac{\partial T}{\partial \tau}
\end{array}\right],
$$

where $\omega=\frac{\Omega}{\epsilon^{2}}$. Since the boundaries are considered to be impermeable and isothermal, therefore we write the boundary condition to solve Eqs. (2.21) as:

$$
\begin{array}{lll}
\psi=0, & T=0 & \text { on } z=0, \\
\psi=0, & T=0 & \text { on } z=1 .
\end{array}
$$

\section{Finite Amplitude Equation and Heat Transport for Stationary Instability}

We now introduce the following asymptotic expansion in Eqs. (2.21):

$$
R a=R_{0 \mathrm{c}}+\epsilon^{2} R_{2}+\epsilon^{4} R_{4}+\ldots,
$$




$$
\begin{aligned}
& \psi=\epsilon \psi_{1}+\epsilon^{2} \psi_{2}+\epsilon^{3} \psi_{3}+\ldots, \\
& T=\epsilon T_{1}+\epsilon^{2} T_{2}+\epsilon^{3} T_{3}+\ldots,
\end{aligned}
$$

where $R_{0 \mathrm{c}}$ is the critical value of the Rayleigh number at which the onset of convection takes place in the absence of gravity modulation. After arranging the systems at different orders of $\epsilon$, we get the following system at the lowest order

$$
\left[\begin{array}{cr}
\nabla_{\xi}^{2} & R_{0 \mathrm{c}} \frac{\partial}{\partial x} \\
-\frac{\mathrm{d} T_{\mathrm{b}}}{\mathrm{d} z} \frac{\partial}{\partial x}-\left(\nabla_{\eta}^{2}+R_{i}\right)
\end{array}\right]\left[\begin{array}{l}
\psi_{1} \\
T_{1}
\end{array}\right]=\left[\begin{array}{l}
0 \\
0
\end{array}\right] .
$$

The solution of the lowest order system subject to the boundary conditions (2.22) is

$$
\begin{aligned}
& \psi_{1}=A(\tau) \sin \left(k_{\mathrm{c}} x\right) \sin (\pi z), \\
& T_{1}=\frac{4 \pi^{2} k_{\mathrm{c}}}{\delta_{R}^{2}\left(R_{i}-4 \pi^{2}\right)} A(\tau) \cos \left(k_{\mathrm{c}} x\right) \sin (\pi z)
\end{aligned}
$$

where $\delta_{R}^{2}=\eta k_{\mathrm{c}}^{2}+\pi^{2}-R_{i}$. The critical value of the Rayleigh number and the corresponding wave number for the onset of stationary convection are as given below:

$$
\begin{aligned}
R_{0 \mathrm{c}} & =\frac{\delta_{\xi}^{2} \delta_{R}^{2}\left(4 \pi^{2}-R i\right)}{4 \pi^{2} k_{\mathrm{c}}^{2}}, \\
k_{\mathrm{c}} & =\left[\frac{\pi^{2}\left(\pi^{2}-R i\right)}{\xi \eta}\right]^{1 / 4},
\end{aligned}
$$

where $\delta_{\xi}^{2}=\frac{\pi^{2}}{\xi}+k_{\mathrm{c}}^{2}$. For the system without internal-heating, we get

$$
\begin{aligned}
R_{0 \mathrm{c}} & =\frac{\delta_{\xi}^{2} \delta_{R}^{2}}{k_{\mathrm{c}}^{2}}, \\
k_{\mathrm{c}} & =\frac{\pi}{(\xi \eta)^{1 / 4}},
\end{aligned}
$$

which is the classical results of Epherre (1975). If we take $\xi=1, \eta=1$, then we get the classical results of Lapwood (1948) for isotropic porous media.

At the second order, we have

$$
\left[\begin{array}{cr}
\nabla_{\xi}^{2} & R_{0 \mathrm{c}} \frac{\partial}{\partial x} \\
-\frac{\mathrm{d} T_{\mathrm{b}}}{\mathrm{d} z} \frac{\partial}{\partial x} & -\left(\nabla_{\eta}^{2}+R_{i}\right)
\end{array}\right]\left[\begin{array}{l}
\psi_{1} \\
T_{1}
\end{array}\right]=\left[\begin{array}{l}
R_{21} \\
R_{22}
\end{array}\right],
$$

where

$$
\begin{aligned}
& R_{21}=0, \\
& R_{22}=\frac{\partial \psi_{1}}{\partial x} \frac{\partial T_{1}}{\partial z}-\frac{\partial \psi_{1}}{\partial z} \frac{\partial T_{1}}{\partial x} .
\end{aligned}
$$

The second-order solution, subject to the boundary condition (2.22), can be obtained as follows:

$$
\begin{aligned}
& \psi_{2}=0 \\
& T_{2}=-\frac{2 \pi^{3} k_{\mathrm{c}}^{2} A^{2}(\tau)}{\delta_{R}^{2}\left(4 \pi^{2}-R_{i}\right)^{2}} \sin (2 \pi z) .
\end{aligned}
$$


The horizontally averaged Nusselt number, $N u$, for the stationary convection(the mode considered in this problem) is given by:

$$
N u(\tau)=1+\frac{\left[\frac{k_{\mathrm{c}}}{2 \pi} \int_{0}^{\frac{2 \pi}{k_{\mathrm{c}}}}\left(\frac{\partial T_{2}}{\partial z}\right) \mathrm{d} x\right]_{z=0}}{\left[\frac{k_{\mathrm{c}}}{2 \pi} \int_{0}^{\overline{k_{\mathrm{c}}}}\left(\frac{\mathrm{d} T_{\mathrm{b}}}{\mathrm{d} z}\right) \mathrm{d} x\right]_{z=0}} .
$$

One can notice here that the gravity modulation is effective at $O\left(\epsilon^{2}\right)$ and affects $N u(\tau)$ through $A(\tau)$ as shown next. Substituting expressions (2.17) and (3.12) in the above expression (3.13) and simplifying, we get

$$
N u(\tau)=1+\frac{4 \pi^{4} k_{\mathrm{c}}^{2} \sin \sqrt{R_{i}}}{\delta_{R}^{2}\left(4 \pi^{2}-R i\right)^{2} \sqrt{R_{i}} \cos \sqrt{R_{i}}}[A(\tau)]^{2} .
$$

At the third order, we have

$$
\begin{aligned}
& {\left[\begin{array}{cr}
\nabla_{\xi}^{2} & R_{0 \mathrm{c}} \frac{\partial}{\partial x} \\
-\frac{\mathrm{d} T_{\mathrm{b}}}{\mathrm{d} z} \frac{\partial}{\partial x} & -\left(\nabla_{\eta}^{2}+R_{i}\right)
\end{array}\right]\left[\begin{array}{l}
\psi_{1} \\
T_{1}
\end{array}\right]=\left[\begin{array}{l}
R_{31} \\
R_{32}
\end{array}\right], } \\
R_{31}= & -\frac{1}{V a} \frac{\partial}{\partial \tau}\left(\nabla_{1}^{2} \psi_{1}\right)-\delta_{1} \cos (\omega \tau) R_{0 \mathrm{c}} \frac{\partial T_{1}}{\partial x}-R_{2} \frac{\partial T_{1}}{\partial x}, \\
R_{32}= & \frac{\partial \psi_{1}}{\partial x} \frac{\partial T_{2}}{\partial z}-\frac{\partial T_{1}}{\partial \tau} .
\end{aligned}
$$

Substituting $\psi_{1}, T_{1}$ and $T_{2}$ from Eqs. (3.3), (3.4), and (3.12) into Eqs. (3.16a) and (3.16b), the expressions for $R_{31}$ and $R_{32}$ can be obtained easily.

Now applying the solvability condition for the existence of third order solution, we get the Ginzburg-Landau equation for stationary convection with time-periodic coefficients in the form:

$$
\left[\frac{\delta^{2}}{\operatorname{Va} \delta_{\xi}^{2}}+\frac{1}{\delta_{R}^{2}}\right] \frac{\mathrm{d} A(\tau)}{\mathrm{d} \tau}=\left[\frac{R_{2}}{R_{0 \mathrm{c}}}+\delta_{1} \cos (\omega \tau)\right] A(\tau)-\frac{\pi^{2} k_{\mathrm{c}}^{2}}{2 \delta_{R}^{2}\left(4 \pi^{2}-R_{i}\right)} A^{3}(\tau) .
$$

The Ginzburg-Landau equation given by (3.17) is the Bernoulli equation and obtaining its analytic solution is difficult due to its non-autonomous nature. Therefore it has been solved numerically using the in-built function NDSolve of Mathmatica 8.0, subject to the suitable initial condition $A(0)=a_{0}$, where $a_{0}$ is the chosen initial amplitude of convection. In our calculations we may assume $R_{2}=R_{0}$, to keep the parameters to the minimum.

\section{Results and Discussion}

In this paper, we study the combined effect of internal heating and gravity modulation on thermal instability in a fluid saturated closely packed anisotropic porous medium. A weakly nonlinear stability analysis has been performed to investigate the effect of gravity modulation on heat transport. The effect of gravity modulation on the Bénard-Darcy system has been assumed to be of order $O\left(\epsilon^{2}\right)$. This means, we consider only small amplitude gravity modulation. Such assumption will help us in obtaining the amplitude equation of convection in 
rather simple and elegant manner and is much easier to obtain than in the case of the Lorenz model.

Before discussing the results obtained in the analysis, we would like to make some comments on the various aspects of the problem, such as:

1. The need for nonlinear stability analysis.

2. The relation of the problem to a real application.

3. The selection of all dimensionless parameters utilized in computations.

4. Retaining the local acceleration term $\frac{1}{V a} \frac{\partial q}{\partial t}$ in the momentum equation.

5. Consideration of numerical values for different parameters.

It is imperative to make a nonlinear study of the problem if one wants to obtain heat transport, which can not be obtained using the linear stability theory, as mentioned in the second last paragraph of the introduction.

External regulation of convection is important in the study of thermal instability in porous media. In this paper, we have considered gravity modulation and internal-heating for either enhancing or inhibiting convective heat transport as is required by a real application.

The parameters that arise here in the study of thermal instability, and influence the heat transport are $V a, R_{i}, \xi, \eta, \delta_{1}, \omega$. The first four are related to the properties of fluid and porous media, and last two concerns the external mechanism that is gravity modulation for controlling the convection.

As pointed out by Vadász (1998) in unmodulated case that there are many real situations in which the value of $V a$ is very large, therefore one can neglect the time-derivative term in Darcy equation (2.15). Further he points out that there are, however, some modern porous medium applications, such as mushy layer in solidification of binary alloys and fractured porous medium, where the value of $V a$ may be considered of the order unity, therefore the time-derivative term in this study has been retained. This is the reason that we have kept the values of $V a$ around one in our calculations, and retained the local acceleration term $\frac{1}{V a} \frac{\partial q}{\partial t}$.

The values of $R_{i}$ are considered to be moderate so that it will not affect the effect of gravity modulation of the system by dominating it otherwise. The values of $\delta_{1}$ is considered to be between 0 and 0.5 , since we are studying the effect of small amplitude modulation on the heat transport. Further, as the effect of low frequencies on the onset of convection as well as on the heat transport is maximum, therefore the modulation of gravity is assumed to be of low frequency.

The numerical results for $N u$ obtained from the expression (3.14) by solving the amplitude equation (3.17) have been presented in the Figs. 2, 3, 4, 5, 6, 7, 8. It can be seen clearly from Eq. (3.14) in conjunction with (3.17) that $N u(\tau)$ is a function of Vadász number $V a$, thermo-mechanical anisotropy parameters $\xi$ and $\eta$, internal heating parameter $R_{\mathrm{i}}$, frequency of modulation $\omega$ and amplitude of modulation $\delta_{1}$.

The effect of gravity modulation on heat transport is shown in Figs. 2, 3, 4, 5, 6, 7, 8, where the graphs for the Nusselt number $N u$ are drawn with respect to time $\tau$. From the figures, it is found that the effect of gravity modulation on the onset of convection is destabilizing, i.e., heat transport and so the Nusselt number is more in this case than in the absence of gravity modulation, which is also clear from the studies of Yang (1997), Malashetty and Padmavathi (1997), and Malashetty and Basavaraj (2002). It is observed that initially, the value of $N u$ starts with 1 , remains constant for some time, thus showing the conduction state, increases with time $\tau$ for intermediate values of $\tau$, thus showing the convection state, and finally becomes constant on further increasing $\tau$, thus achieving the steady state. The behaviour of $N u$ becomes oscillatory for intermediate values of time $\tau$. 


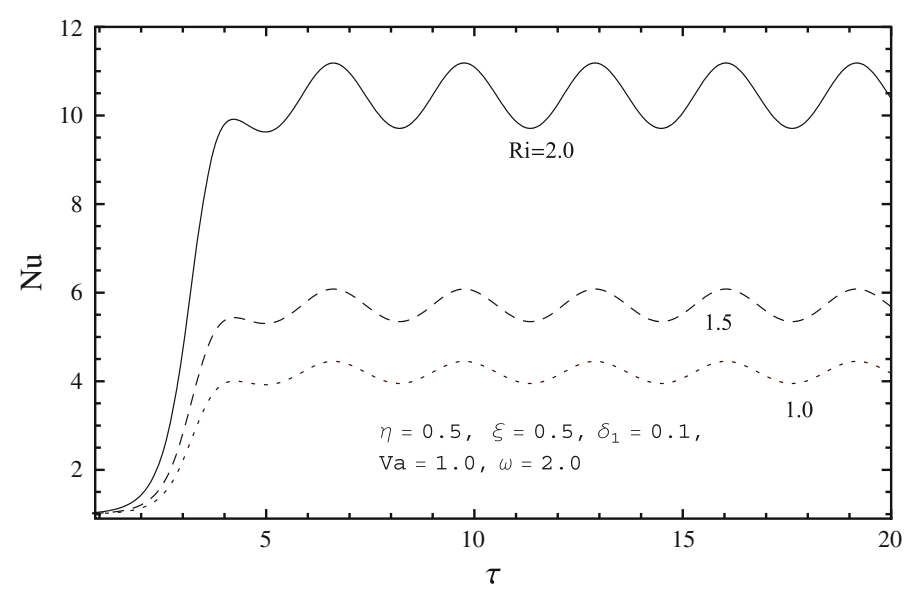

Fig. $2 N u$ vs. time $\tau$, for different values of $R_{\mathrm{i}}$

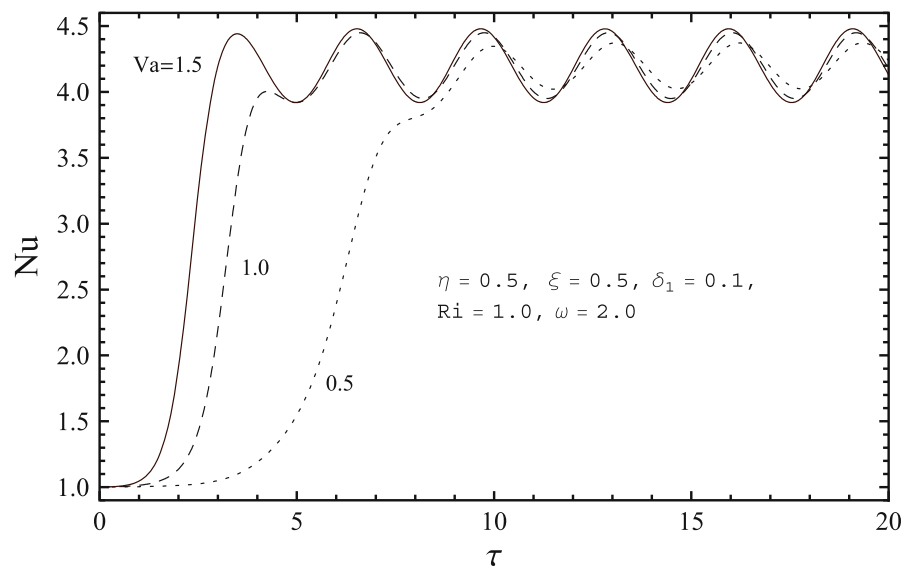

Fig. $3 N u$ vs. time $\tau$, for different values of $V a$

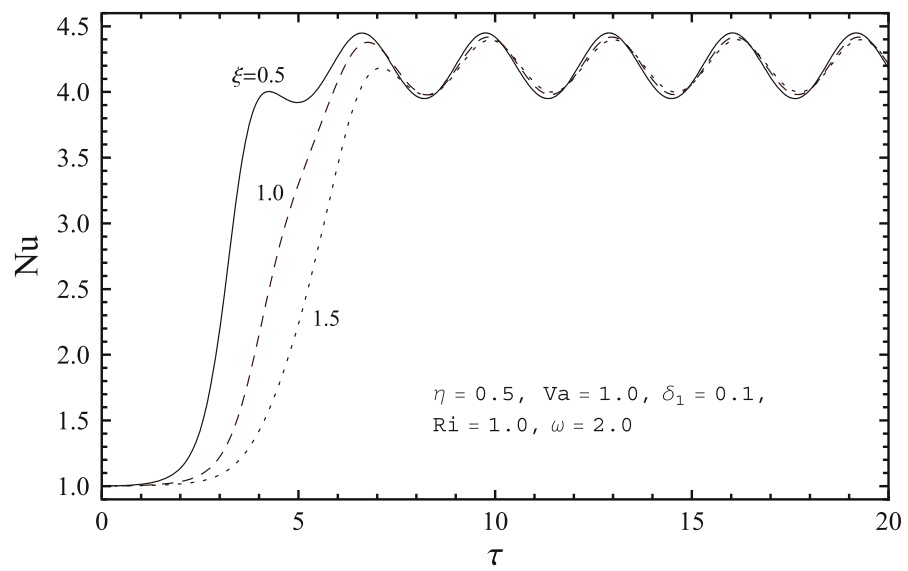

Fig. $4 N u$ vs. time $\tau$ for different values of $\xi$ 


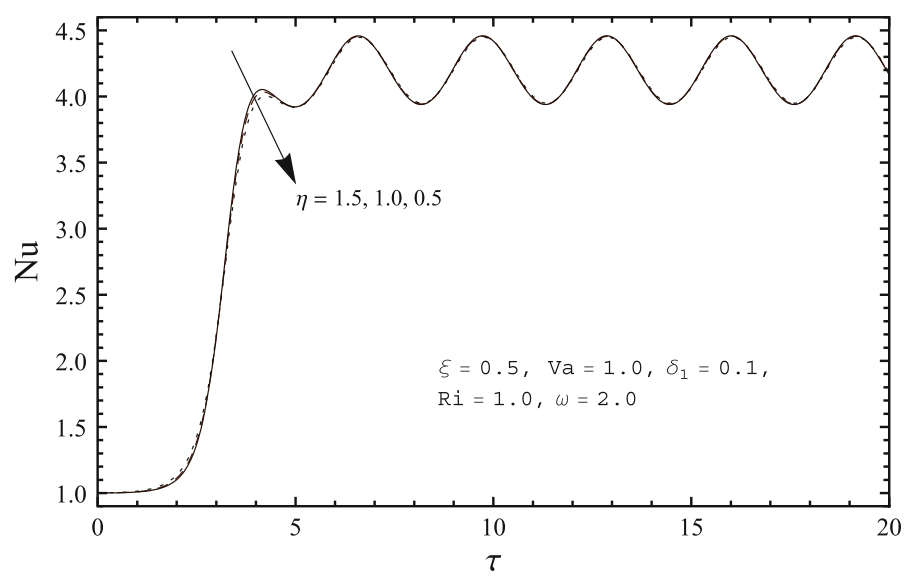

Fig. $5 N u$ vs. time $\tau$ for different values of $\eta$

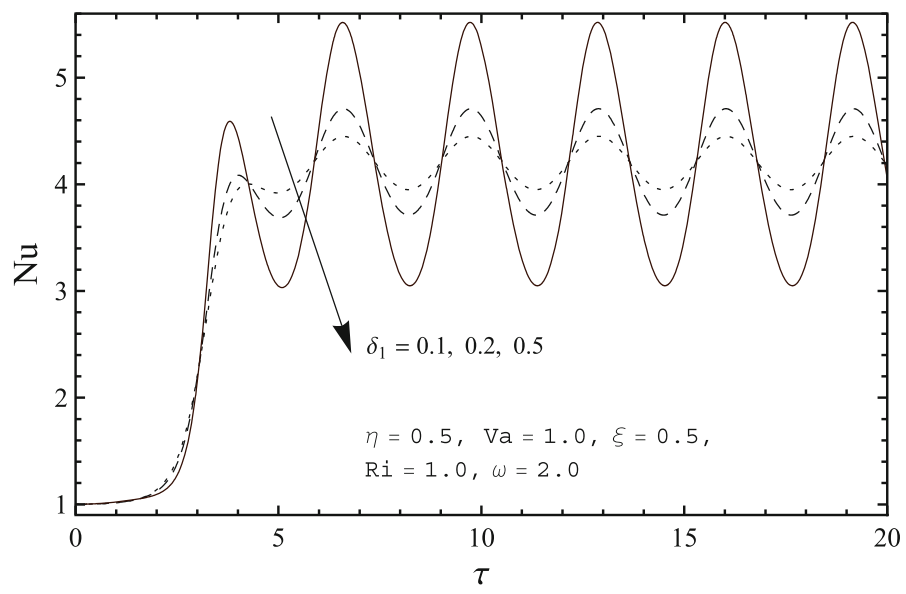

Fig. $6 N u$ vs. time $\tau$ for different values of $\delta_{1}$

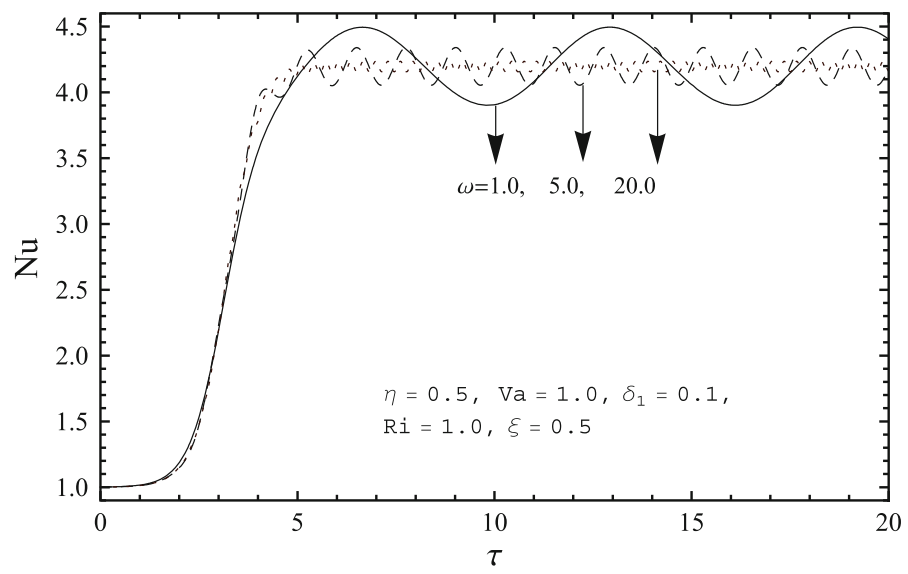

Fig. $7 N u$ vs. time $\tau$ for different values of $\omega$ 


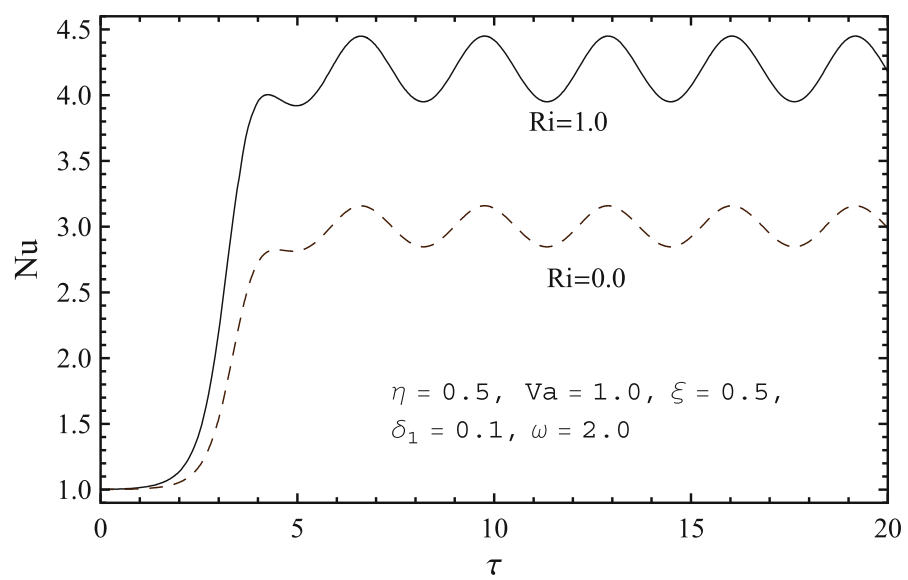

Fig. $8 N u$ vs. time $\tau$. Comparison between internal- and non-internal-heating cases

From Fig. 2, we observe that the effect of internal heating on thermal instability is destabilizing, as heat transport increases on increasing $R_{\mathrm{i}}$. The heat transport is more at higher values of $R_{\mathrm{i}}$. This confirms the results obtained most recently by Bhadauria (2012) and Bhadauria et al. (2011). Effect of Vadász number $V a$ on the system is also destabilizing (Fig. 3) as heat transport increases on increasing its value. This result is compatible with the result of Vadász (1998) obtained for rotating porous medium. From Fig. 4, we find that an increment in $\xi$ decreases heat transport, thus suppresses the convection. When $\xi$ increases, then either $K_{x}$ increases or $K_{z}$ decreases, and so in both these cases fluid flow through porous medium decreases in vertical direction in comparison to the flow in horizontal direction. This delays the convection, and thus decreases the heat transport in the system. However, the effects of $V a$ and $\xi$ on heat transport diminish at higher values of time $\tau$. Further, it is found from Fig. 5 that on increasing the value of thermal anisotropy $\eta$, heat transport decreases initially, and then increases as time passes. Thus the effect of mechanical and thermal anisotropies is found to be opposite at large time, compatible with the results of Epherre (1975), Kuznetsov and Nield (2008), and Bhadauria (2012), obtained for unmodulated case. However at small time $\tau$, the effect of thermal anisotropy $\eta$ is similar to $\xi$ which is just opposite to the unmodulated case. This may be due to the gravity modulation effect.

From the results of Nield and Kuznetsov (2007), it was found that Rayleigh number increases on increasing the value of $\eta$, that is the onset of convection takes place at later point, thus an increment in $\eta$ stabilizes the system so the heat transport is less. This result is qualitatively similar to the present result given in Fig. 5, where the value of $N u$ decreases initially on increasing the value of $\eta$ thus stabilizing the system. Further in Figs. 4 and 5, we find certain values of time $\tau$, where the value of $N u$ is found to decrease on increasing $\eta$ and decreasing $\xi$, which is same as stabilizing effects given in Nield and Kuznetsov (2007).

The effect of $\delta_{1}$ on $N u$ is depicted in the Fig. 6. From the figure we observe that effect of increasing the amplitude of gravity modulation is to increase the heat transport, thus advancing the convection. Further, from Fig. 7, we find that an increment in the frequency of modulation decreases the magnitude of $N u$, and shortens the wavelength of oscillations. As the frequency increases from 1 to 20, the magnitude of $N u$ decreases, and the effect of 

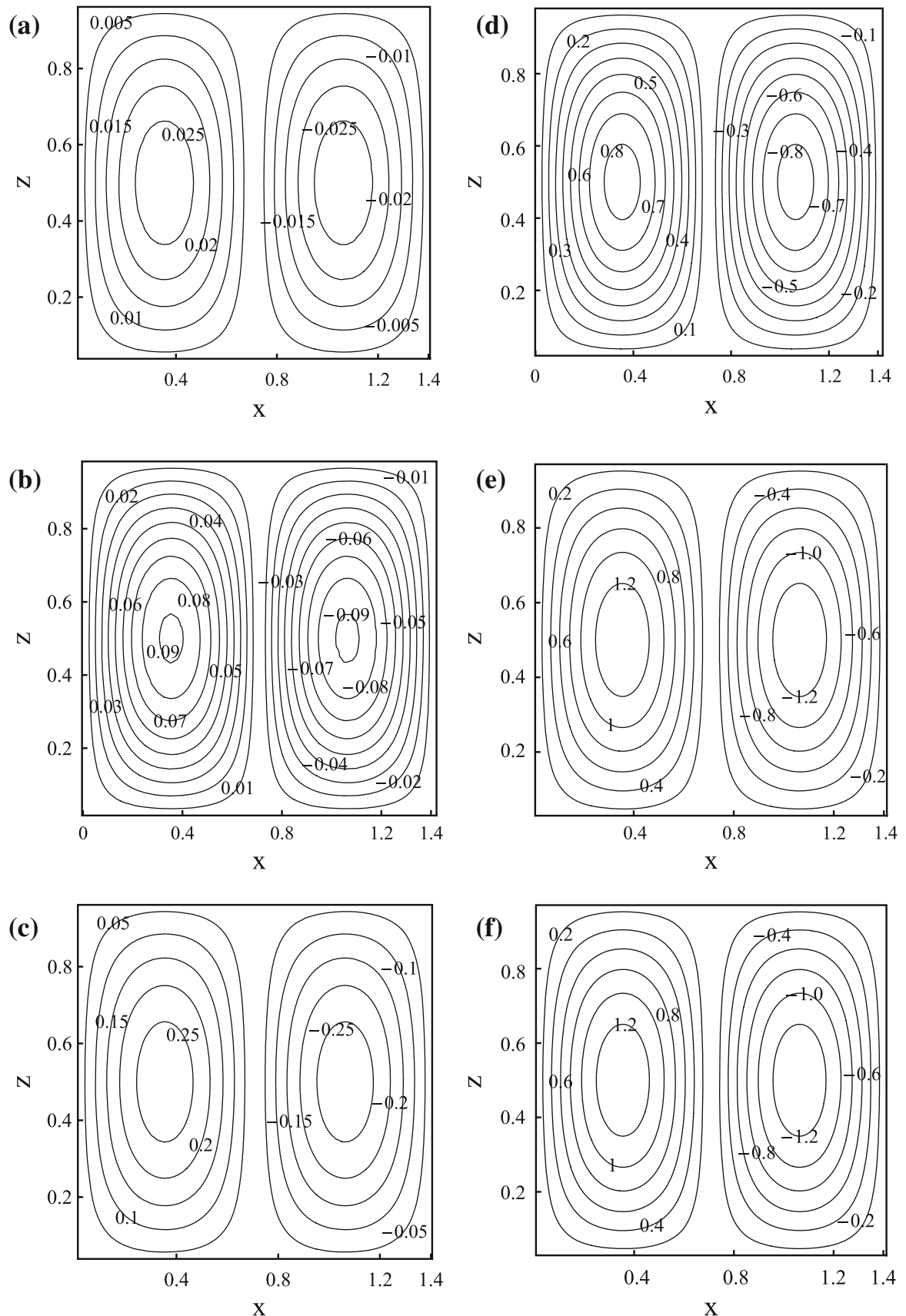

Fig. 9 Streamlines at (a) $\tau=0.1$, (b) $\tau=1.0$, (c) $\tau=2.0$, (d) $\tau=3.0$, (e) $\tau=4.0,(\mathbf{f}) \tau=5.0$

modulation on heat transport diminishes. On further increasing the value of $\omega$ that is at $\omega=50$, the effect of gravity modulation on thermal instability disappears altogether. This result is very much in agreement with the linear studies of Yang (1997), Malashetty and 
(a)

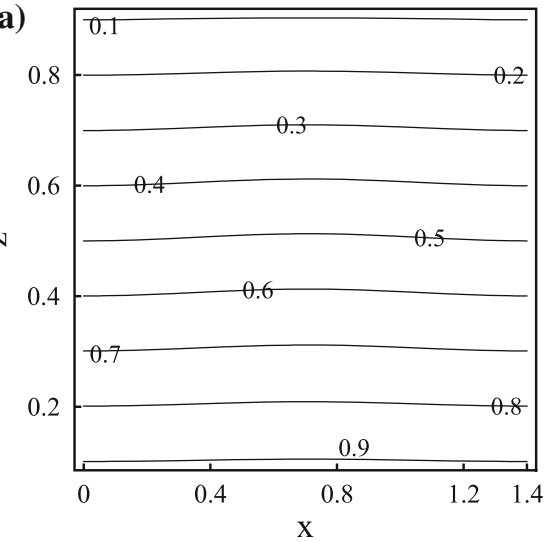

(b)

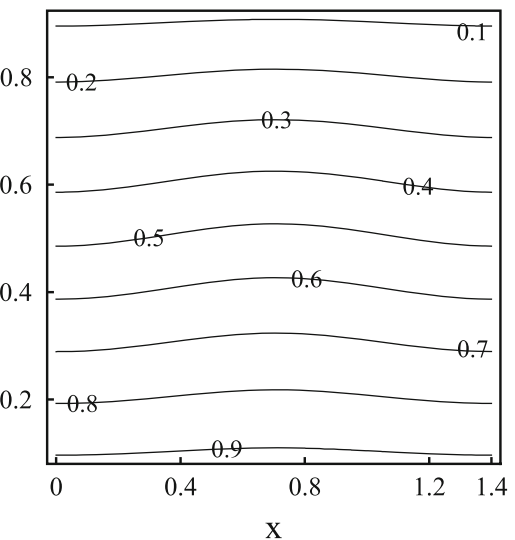

(c)

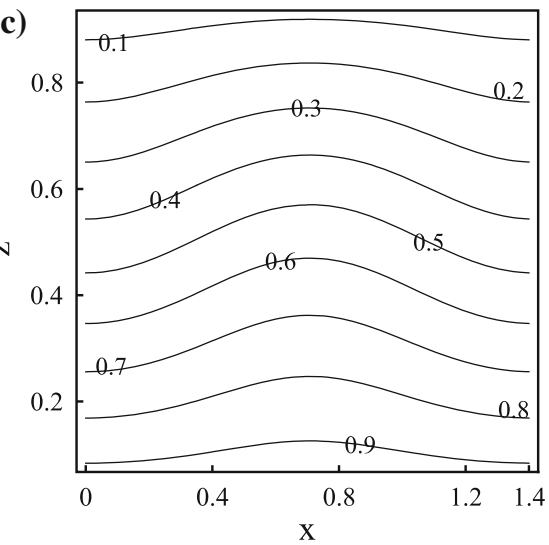

(c)

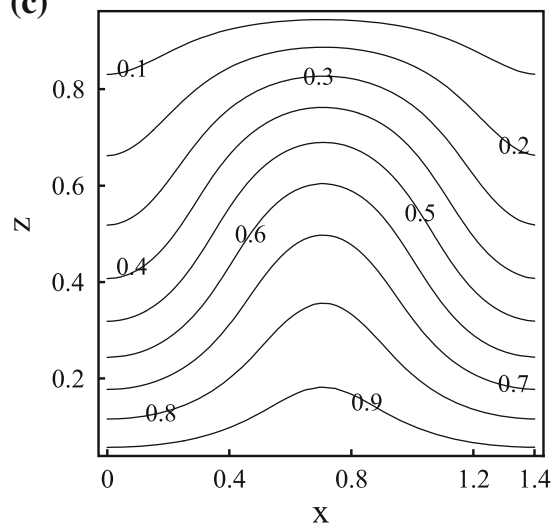

(d)

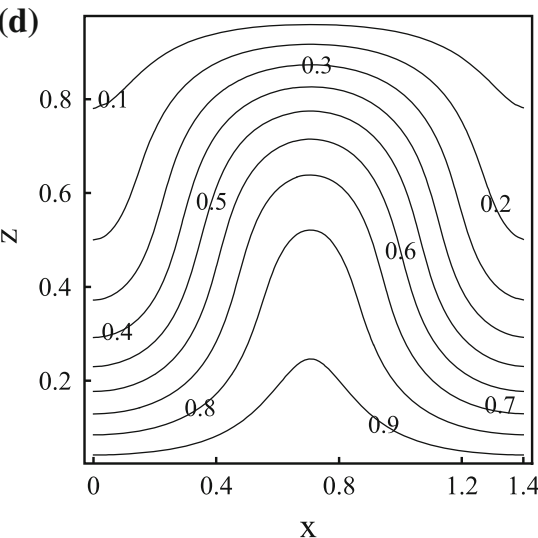

(e)

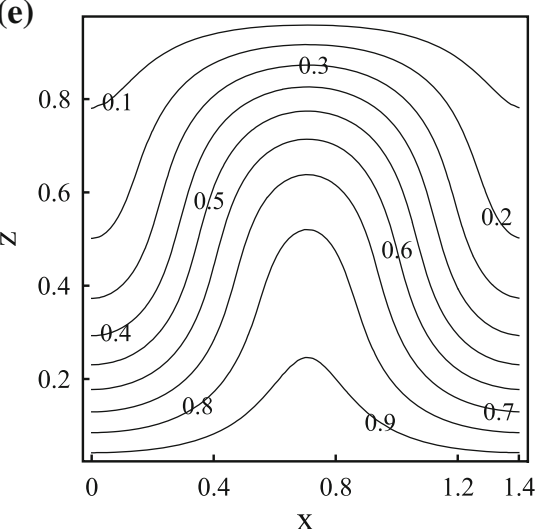

Fig. 10 Isotherms at (a) $\tau=0.1$, (b) $\tau=1.0$, (c) $\tau=2.0$, (d) $\tau=3.0$, (e) $\tau=4.0$, (f) $\tau=5.0$

Padmavathi (1997), and Malashetty and Basavaraj (2002), where the correction in the critical value of Rayleigh number due to gravity modulation becomes almost zero at higher frequencies, and thus no effect of gravity modulation. 
The present result of internal heating has been compared with the results of non-internal heating in Fig. 8. We observe that in case of internal heating of the system, the heat transport in the system is more than that in the absence of internal heating, thus internal heating advances the onset of convection.

In Figs. 9 and 10, the streamlines and the corresponding isotherms are depicted respectively, at $\tau=0.1,1.0,2.0,3.0,4.0$, and 5.0 for $R_{i}=0.1, \mathrm{Va}=1.0, \xi=0.5$, $\eta=0.5, \delta_{1}=0.1, \omega=2$ and $\epsilon=0.5$. From the figures, we observe that initially when time $\tau$ is small the magnitude of streamlines is also small, and isotherms are straight that is the system is in conduction state. However, as time increases the magnitude of streamlines increases and the isotherms loses their evenness. This shows that now convection is taking place in the system. Convection becomes faster on further increasing the value of time $\tau$. Further from Figs.9d, e and 10d, e, it is found that the system achieves its steady state at about $\tau=5.0$ as their is no change in the streamlines and isotherms on further increasing $\tau$ beyond 5.0.

\section{Conclusions}

In the present paper, we consider the combined effect of internal heating and gravity modulation on Bénard-Darcy convection in a closely packed porous medium, and perform a weak non-linear stability analysis by using the Gingburg-Landau equation. The following conclusions are drawn:

1. Effect of gravity modulation is oscillatory on heat transport in the system.

2. The effects of internal heating and $V a$ are to increase the heat transport in the system.

3. The effect of an increment in the frequency of modulation is to decrease the heat transport, while amplitude of modulation increases the heat transport.

4. Heat transport decreases on increasing the mechanical anisotropy parameter $\xi$.

5. Effect of increasing the value of thermal anisotropy parameter $\eta$ is negligible on heat transport.

6. Heat transport is more in this case than in the absence of internal heating.

7. As time $\tau$ increases, the magnitude of streamlines increases and the isotherms loses their evenness.

8. Steady state is attained by the system at $\tau=5.0$.

Further, we have

1. $N u / R_{\mathrm{i}}=1.0<N u / R_{\mathrm{i}}=1.5<N u / R_{\mathrm{i}}=2.0$,

2. $N u / V a=0.5<N u / V a=1.0<N u / V a=1.5$,

3. $N u / \xi=1.5<N u / \xi=1.0<N u / \xi=0.5$,

4. $N u / \delta_{1}=0.1<N u / \delta_{1}=0.2<N u / \delta_{1}=0.5$,

5. $N u / \omega=20.0<N u / \omega=5<N u / \omega=1.0$,

6. $N u / R_{\mathrm{i}}=0.0<N u / R_{\mathrm{i}}>0$.

Acknowledgments This study was done during the visit of the author B.S. Bhadauria (BSB) to the Universiti Kebangsaan Malaysia (UKM), in June, 2012 as visiting Professor of Mathematics. The author BSB gratefully acknowledges the grant provided by UKM out of the University Research Fund OUP-2012-61. The author BSB is also grateful to Banaras Hindu University, Varanasi for sanctioning the lien to work as Professor of Mathematics at Department of Applied Mathematics, BB Ambedkar University, Lucknow, India. 


\section{References}

Bhadauria, B.S., Anoj, K., Jogendra, K., Sacheti, N.C., Pallath, C.: Natural convection in a rotating anisotropic porous layer with internal heat generation. Transp. Porous Media 90, 687-705 (2011)

Bhadauria, B.S.: Double diffusive convection in a saturated anisotropic porous layer with internal heat source. Trans. Porous Media 92, 299-320 (2012)

Bhattacharya, S.P., Jena, S.K.: Thermal instability of a horizontal layer of micropolar fluid with heat source. Proc. Indian Acad. Sci. (Math. Sci.) 93(1), 13-26 (1984)

Epherre, J.F.: Crit'ere d'apparition de la convection naturalle dans une couche poreuse anisotrope. Rev. Gén. Therm. 168, 949-950 (1975) (English translation, Int. Chem. Engng. 17, 615-616 (1977))

Govender, S.: Stability of convection in a gravity modulated porous layer heated from below. Transp. Porous Media 57(1), 113-123 (2004)

Govender, S.: Linear stability and convection in a gravity modulated porous layer heated from below-transition from synchronous to subharmonic solutions. Transp. Porous Media 59(2), 227-238 (2005)

Govender, S.: Weak non-linear analysis of convection in a gravity modulated porous layer. Transp. Porous Media 60, 33-42 (2005)

Haajizadeh, M., Ozguc, A.F., Tien, C.L.: Natural convection in a vertical porous enclosure with internal heat generation. Int. J. Heat Mass Transf. 27, 1893-1902 (1984)

Herron Isom, H.: Onset of convection in a porous medium with internal heat source and variable gravity. Int. J. Eng. Sci. 39, 201-208 (2001)

Ingham, D.B., Pop, I.: Transport phenomena in porous media. Pergamon, Oxford (1998)

Ingham, D.B., Pop, I.: Transport Phenomena in Porous Media, vol. III. Elsevier, Oxford (2005)

Joshi, M.V., Gaitonde, U.N., Mitra, S.K.: Analytical study of natural convection in a cavity with volumetric heat generation. ASME J. Heat Transf. 128, 176-182 (2006)

Khalili, A., Huettel, M.: Effects of throughflow and internal heat generation on convective instabilities in an anisotropic porous layer. J. Porous Media 5(3), 64-75 (2002)

Kuznetsov, A.V., Nield, D.A.: The effects of combined horizontal and vertical heterogenity on the onset of convection in a porous medium: double diffusive case. Transp. Porous Media 72, 157-170 (2008)

Kuznetsov, A.V.: The onset of bioconvection in a suspension of negatively geotactic microorganisms with high-frequency vertical vibration. Int. Comm. Heat Mass Transf. 32, 1119-1127 (2005)

Kuznetsov, A.V.: Linear stability analysis of the effect of vertical vibration on bioconvection in a horizontal porous layer of finite depth. J. Porous Media 9, 597-608 (2006)

Kuznetsov, A.V.: Investigation of the onset of bioconvection in a suspension of oxytactic microorganisms subjected to high frequency vertical vibration. Theor. Comput. Fluid Dynam. 20, 73-87 (2006)

Lapwood, E.R.: Convection of a fluid in porous medium. Proc. Camb. Phil. Soc. 44, 508-521 (1948)

Malashetty, M.S., Padmavathi, V.: Effect of gravity modulation on the onset of convection in a fluid and porous layer. Int. J. Eng. Sci. 35, 829-839 (1997)

Malashetty, M.S., Basavaraj, D.: Rayleigh-Bénard convection subject to time-dependent wall temperature/ gravity in a fluid saturated anisotropic porous medium. Heat Mass Transf. 38, 551-563 (2002)

Malashetty, M.S., Swamy, M.: Effect of gravity modulation on the onset of thermal convection in rotating fluid and porous layer. Phys. Fluids 23(6), 064108 (2011)

Nield, D.A., Bejan, A.: Convection in Porous Media. 3rd edn. Springer, New York (2006)

Nield, D.A., Kuznetsov, A.V.: The effects of combined horizontal and vertical heterogeneity and anisotropy on the onset of convection in a porous medium. Int. J. Thermal Sci. 46, 1211-1218 (2007)

Parthiban, C., Patil, P.R.: Thermal instability in an anisotropic porous medium with internal heat source and inclined temperature gradient. Int. Comm. Heat Mass Transf. 24(7), 1049-1058 (1997)

Rajagopal, K.R., Saccomandib, G., Vergoric, L.: A systematic approximation for the equations governing convection-diffusion in a porous medium. Nonlinear Anal. Real World Appl. 11(4), 2366-2375 (2010)

Rao, Y.F., Wang, B.X.: Natural convection in vertical porous enclosures with internal heat generation. Int. J. Heat Mass Transf. 34, 247-252 (1991)

Razi, Y.P., Mojtabi, I., Charrier-Mojtabi, M.C.: A summary of new predictive high frequency thermo-vibrational modes in porous media. Transp. Porous Media 77, 207-208 (2009)

Rees, D.A.S., Pop, I.: The effect of G-jitter on vertical free convection boundary-layer flow in porous media. Int. Comm. Heat Mass Transf. 27(3), 415-424 (2000)

Rees, D.A.S., Pop, I.: The effect of G-jitter on free convection near a stagnation point in a porous medium. Int. J. Heat Mass Transf. 44, 877-883 (2001)

Rees, D.A.S., Pop, I.: The effect of large-amplitude G-jitter vertical free convection boundary-layer flow in porous media. Int. J. Heat Mass Transf. 46, 1097-1102 (2003)

Rionero, S., Straughan, B.: Convection in a porous medium with internal heat source and variable gravity effects. Int. J. Eng. Sci. 28(6), 497-503 (1990) 
Saravanan, S., Arunkumar, A.: Convective instability in a gravity modulated anisotropic thermally stable porous medium. Int. J. Engg. Sci. 48, 742-750 (2010)

Saravanan, S., Purusothaman, A.: Floquent instability of a modulated Rayleigh-Bénard problem in an anisotropic porous medium. Int. J. Therm. Sci. 48, 2085-2091 (2009)

Saravanan, S., Sivakumar, T.: Onset of filteration convection in a vibrating medium: The Brinkman model. Phys. Fluids 22, 034104 (2010)

Saravanan, S., Sivakumar, T.: Thermovibrational instability in a fluid saturated anisotropic porous medium. ASME J. Heat Transf. 133, 051601.1-051601.9 (2011)

Siddhavaram, V.K., Homsy, G.M.: The effects of gravity modulation on fluid mixing Part 1 Harmonic modulation. J. Fluid Mech. 562, 445-475 (2006)

Siddheshwar, P.G., Vanishree, R.K., Melson, A.C.: Study of heat transport in Bénard-Darcy convection with g-jitter and thermomechanical anisotropy in variable viscosity liquids. Transp. Porous Media 92(2), 277$288(2012)$

Strong, N.: Effect of vertical modulation on the onset of filtration convection. J. Math. Fluid Mech. 10, 488502 (2008)

Strong, N.: Double-diffusive convection in a porous layer in the presence of vibration. SIAM J. Appl. Math. 69, 1263-1276 (2008)

Siddheshwar, P.G., Bhadauria, B.S., Srivastava, Alok.: An analytical study of nonlinear double diffusive convection in a porous medium under temperature/gravity modulation. Transp. Porous Media 91, 585604 (2012)

Straughan, B.: The Energy Method, Stability, and Nonlinear Convection-Second edition, Appl. Math. Sci. Ser.. Springer, New York (2004)

Vadász, P.: Coriolis effect on gravity-driven convection in a rotating porous layer heated from below. J. Fluid Mech. 376, 351-375 (1998)

Vadász, P. (ed.): Emerging Topics in Heat and Mass Transfer in Porous Media . Springer, New York (2008)

Vafai, K. (ed.): Handbook of Porous Media. Marcel Dekker, New York (2000)

Vafai, K. (ed.): Handbook of Porous Media. Taylor and Francis (CRC), Boca Raton (2005)

Vanishree, R.K.: Combined effect of temperature and gravity modulations on the onset of convection in an anisotropic porous medium. Int. J. Appl. Mech. Engg. 15(1), 267-291 (2010)

Yang, Wen-Mei.: Stability of viscoelastic fluids in a modulated gravitational field. Int. J. Heat Mass Transf. 40(6), 1401-1410 (1997) 